\title{
Captures
}

Figures, théories et pratiques de l'imaginaire

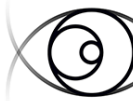

C A P T U U R E S

Quelques lignes « intra muros et extra » Lecture graphique de l'incipit du Père Goriot de Balzac

\section{Véronique Cnockaert}

Volume 2, Number 2, 2017

Imaginaire de la ligne

URI: https://id.erudit.org/iderudit/1059772ar

DOI: https://doi.org/10.7202/1059772ar

See table of contents

Publisher(s)

Figura, Centre de recherche sur le texte et l'imaginaire

ISSN

2371-1930 (digital)

Explore this journal

Cite this article

Cnockaert, V. (2017). Quelques lignes " intra muros et extra " : lecture graphique de l'incipit du Père Goriot de Balzac. Captures, 2(2).

https://doi.org/10.7202/1059772ar
Article abstract

The first words of Balzac's Le Père Goriot present a remarkable criss-crossing of distinct lines that never cease to echo one another. Graphic line, historic line, geographic line, institutional line, and cultural line intersect, creating a tight-wound tangle offering up a rich scriptural invention that is analyzed in this article.
This document is protected by copyright law. Use of the services of Erudit (including reproduction) is subject to its terms and conditions, which can be viewed online.

https://apropos.erudit.org/en/users/policy-on-use/ 
Version enrichie de cet article : http://revuecaptures.org/node/952

\section{Quelques lignes « intra muros et extra»}

\section{Lecture graphique de l'incipit du Père Goriot de Balzac}

\section{Véronique Cnockaert}

Résumé :

L'incipit du Père Goriot de Balzac présente un entrecroisement remarquable de lignes distinctes qui ne cessent d'être l'écho l'une de l'autre. Ligne graphique, ligne historique, ligne géographique, ligne institutionnelle et ligne culturelle forment un écheveau serré, tissage qui offre un mode d'invention scriptural riche en significations que cet article se propose d'analyser.

The first words of Balzac's Le Père Goriot present a remarkable criss-crossing of distinct lines that never cease to echo one another. Graphic line, historic line, geographic line, institutional line, and cultural line intersect, creating a tight-wound tangle offering up a rich scriptural invention that is analyzed in this article.

\section{L'évocation de la pensée par la ligne, l'arabesque \\ et les moyens plastiques, voilà mon but.}

Gustave Moreau1.

Sur un sol parisien connu pour ses accidents, traçons, après Balzac, quatre lignes droites, qui font se rejoindre la Maternité de Port-Royal, l'hospice de la Vieillesse, l'hôpital des Vénériens et celui des Incurables, et qui enclosent une partie du quartier du Val-de-Grâce et du faubourg Saint-Marceau. Ce quartier, que découvrit Jean-Jacques Rousseau en arrivant à Paris et dont il dira dans Les Confessions qu'aucune « magnificence » parisienne n'a jamais réussi à gommer ce premier souvenir d'insalubrité et de pauvreté, à tel point qu'il en gardera « un secret dégoût pour l'habitation de cette capitale 2 » (Rousseau, 1832 [1782]: 187), va néanmoins connaître une gloire toute littéraire grâce au Père Goriot dont il est le théâtre initial. C'est en effet entre ces quatre sommets qui a eux seuls résument les étapes importantes d'une vie (la naissance, la vieillesse et la mort par amour ou par abandon) que se joue le drame d'un père trop amoureux de ses filles et l'ascension sociale d'un jeune homme de province venu faire ses armes à Paris, tous deux logeant dans la pension désormais célèbre, sise rue Neuve-Sainte-Geneviève, de madame Vauquer $\underline{3}$. Si nous avons choisi l'incipit de ce roman, c'est qu'il présente un entrecroisement tout à fait remarquable de lignes distinctes qui ne cessent cependant d'être l'écho l'une de l'autre. Ligne graphique, ligne historique, ligne géographique, ligne institutionnelle et ligne culturelle forment un écheveau serré, tissage qui propose un mode d'invention scriptural riche en significations. 


\section{Ligne descendante}

Ce n'est pas sur une ligne graphique que s'ouvre l'incipit, mais sur une, toute virtuelle, qui appartient au schème vertical de la chute. Ainsi, dès le début, une verticalité descendante emporte avec elle la suite de l'incipit et lui sert de forme structurante. Qu'il s'agisse des personnages ou de l'environnement, tout subit sa logique puissante qui n'épargne rien ni personne :

Cette pension, connue sous le nom de la Maison Vauquer, admet également des hommes et des femmes, des jeunes gens et des vieillards, sans que jamais la médisance ait attaqué les mœurs de ce respectable établissement. Mais aussi depuis trente ans ne s'y était-il jamais vu de jeune personne, et pour qu'un jeune homme y demeure, sa famille doit lui faire une bien maigre pension. Néanmoins en 1819, époque à laquelle ce drame commence, il s'y trouvait une pauvre jeune fille. (Balzac, 2004 [1834]: 46.)

Dans ce premier paragraphe, les individus comme les choses sont frappés du sceau de la déréliction et le mot est à prendre en son sens fort. C'est ainsi pour madame Vauquer, qui a perdu son rang, pour « la jeune fille pauvre », sous-entendu Victorine Taillefer, qui n'a pas été reconnue par son père millionnaire, et pour «le jeune homme » à qui la « famille doit [...] faire une bien maigre pension » pour qu'il demeure dans une telle maison, c'est-à-dire Eugène de Rastignac, dont la particule ne préjuge en rien de la fortune, comme l'indique ici son statut présenté par anticipation. On le voit, les lignes de filiation sont toutes interrompues (par la descendance ruinée de madame Vauquer, par la mort du père pour Victorine, par la volonté — l'histoire nous l'apprendra - de couper avec le sien et avec la province pour Rastignac). Ce nouveau siècle, héritier d'une Révolution qui a coupé la tête du roi, coupe, ainsi que ce début de roman le laisse voir en creux, avec la métrique biologique de l'Ancien Régime, mais pas seulement. La suite du paragraphe révèle que ces revers de fortune ne sont pas uniquement le lot des êtres, ils touchent aussi le roman et plus spécifiquement le « drame » qui, en « ces temps de douloureuse littérature », a perdu en considération.

En quelque discrédit que soit tombé le mot drame par la manière abusive et tortionnaire dont il a été prodigué dans ces temps de douloureuse littérature, il est nécessaire de l'employer ici : non que cette scène soit dramatique dans le sens vrai du mot; mais l'œuvre accomplie, peut-être aura-t-on versé quelques larmes intra muros et extra Sera-t-elle comprise au-delà de Paris? Le doute est permis. (4748.)

Retenons pour l'instant que la dévalorisation générique double la chute sociale des trois premiers protagonistes, mais ce n'est pas tout. Alors que, jusqu'ici, la ligne dominante en décrivait une verticale, l'indication « intra muros et extra» la tord pour former un cercle, celui du mur d'octroi qui ceint Paris depuis 
1784. En insistant sur le dedans et le dehors de la ville, le narrateur laisse entendre que le récit qui va suivre ne peut être compris que de l'intérieur (“Sera-t-elle comprise au-delà de Paris? Le doute est permis. »), centralité du lecteur qui signe pour Balzac celle du roman sur la scène littéraire; on sait que, durant toute sa carrière, le romancier travaillera à anoblir ce genre souverainement bâtard, et il y parviendra. Le Père Goriot ne sera d'ailleurs pas étranger à cette promotion. Seuls donc les Parisiens semblent en mesure de saisir les affres de ce père malade d'amour pour ses filles et les turpitudes d'un jeune homme en quête de reconnaissance. C'est qu'ils connaissent d'expérience cette « illustre vallée de plâtras », cette « vallée remplie de souffrances réelles et de joies souvent fausses », agglomérat de « vices » et de "vertus » qui va des « buttes Montmartre » aux « hauteurs de Montrouge » (Balzac, 2004 [1834]: 48), et où s'entrechoquent violemment crimes et pitié. Paris sous la plume de Balzac s'apparente à une toile arachnéenne qui attrape ceux qui n'en connaissent pas les ruses et les chemins tortueux, comme le confirme le texte : " Le char de la civilisation [...], à peine retardé par un cœur moins facile à broyer que les autres et qui enraye sa route, l'a brisé bientôt et continue sa marche glorieuse » (48). Ainsi, aux « ruisseaux noirs de boue » (48) qui traversent la ville et la maculent, se superpose le tracé de la roue civilisatrice, obscure comme l'encre, sombre comme une gloire volée qui anéantit les cœurs, même les plus coriaces.

\section{Le cercle parisien}

On le voit, le cercle parisien, périmètre matriciel, ne déboute en rien la ligne verticale; au contraire, il la contient comme pour lui permettre de se multiplier et de se métamorphoser sans frein. Et c'est le cas si l'on en juge par la description du quartier de la pension Vauquer. Dès le deuxième paragraphe, la verticalité descendante s'affiche à nouveau, mais cette fois-ci graphiquement en orientant spatialement la description. Elle rejoint par ce mouvement les dérélictions précédentes : "La pension bourgeoise est située dans le bas de la rue NeuveSainte-Geneviève, à l'endroit où le terrain s'abaisse vers la rue de l'Arbalette par unepente si brusque et si rude que les chevaux la montent ou la descendent rarement $\stackrel{4}{\text {. } ~ » ~(B a l z a c, ~} 2004$ [1834]: 49.) Encore une fois, la « pente » est un guide qui mène vers d'insolites noirceurs, puisque l'âme de ceux qui s'y abandonnent se désole, tout comme la lumière décline. Comme dans le paragraphe précédent, rien n'échappe à l'inféodation de cette triste et douloureuse verticalité, au point que la rue, ruinée par ce trait, se transforme en caveau : « de marche en marche, le jour diminue et le chant du conducteur se creuse, alors que le voyageur descend aux Catacombes. Comparaison vraie! Qui décidera de ce qui est plus horrible à voir, ou des cœurs desséchés, ou des crânes vides? » (49.)

Alors que la dénivellation invite aux ruissellement des eaux de la ville, on peut s'étonner de la sècheresse du lieu; il convient d'ailleurs de s'arrêter sur cette anomalie, tant la raison apparaît étonnante. Ainsi, la nature éminemment pentue de l'endroit 
est favorable au silence qui règne dans ces rues serrées entre le dôme du Val-de-Grâce et le dôme du Panthéon, deux monuments qui changent les conditions de l'atmosphère en y jetant des tons jaunes, en y assombrissant tout par les teintes sévères que projettent leurs coupoles. Là, les pavés sont secs, les ruisseaux n'ont ni boue ni eau, l'herbe croît le long des murs. L'homme le plus insouciant s'y attriste comme tous les passants, le bruit d'une voiture y devient un événement, les maisons y sont mornes, les murailles y sentent la prison. Un Parisien égaré ne verrait là que des pensions bourgeoises ou des institutions, de la misère ou de l'ennui, de la vieillesse qui meurt, de la joyeuse jeunesse contrainte à travailler. Nul quartier de Paris n'est plus horrible, ni, disons-le, plus inconnu. (49.)

Enclavé entre ces deux dômes monumentaux, l'endroit subit un assombrissement chromatique et moral. Le plus étrange cependant est que cet obscurcissement résulte de la projection de « tons jaunes », le jaune étant une couleur reconnue pour ses qualités chatoyantes et éclaircissantes. Quelle est donc cette projection sinistrement lumineuse, d'où tient-elle cette capacité à flétrir les lieux qu'elle domine? Revenons pour y répondre aux sources de l'ombre projetée, le dôme du Val-de-Grâce et celui du Panthéon.

L'église du Val-de-Grâce est une ancienne abbaye construite en 1624, sur la demande d'Anne d'Autriche, à laquelle une église entièrement dédiée à la nativité a été greffée en 1645, toujours selon les vœux de la reine, devenue Régente et mère entre temps. Notons que l'église possédait une chapelle dite " chapelle des cœurs » qui renfermait les cœurs embaumés de 45 rois et reines. Ces urnes reliquaires furent vendues ou échangées sous la Révolution. En 1793, la Convention réaffecte l'édifice en hôpital militaire. Ainsi, alors que la vocation initiale du Val-de-Grâce était de célébrer la naissance du Christ et de protéger les âmes et les cœurs, depuis la Terreur, on n'y soigne désormais plus que les corps. Quant au Panthéon, il fut à l'origine, en 1764, une église construite pour abriter la chasse de sainte Geneviève; finalement, en 1791, il se transforme en «Panthéon des grands hommes ". Sous Napoléon $1^{\mathrm{er}}$ et Louis XVIII, l'édifice retrouve pour un temps sa vocation première, mais, en 1830, sous Louis-Philippe, l'église redevient un Panthéon. Lors de l'année de publication du Père Goriot, le Panthéon est donc un temple laïque depuis quatre an૬. Et, même si le récit débute en 1819, il semble, à suivre le texte de Balzac, qu'en ces lieux désolés, tristes et noirs, «l'ancien pouvoir religieux », ainsi que le remarque Paul Bénichou, « est devenu étranger à la société » (1977: 474), et que le cœur sec des hommes a damé le pion aux cœurs embaumés des rois encore bénis des dieux, ce qui, en 1834, on le sait, n'est plus le cas, puisque même la monarchie est constitutionnelle. La ligne descendante accompagne en quelque sorte cette désacralisation des institutions : de l'église à l'hôpital militaire, de l'église au monument laïque, du ciel aux Catacombes.

Sur la rue Neuve-Sainte-Geneviève plane donc l'ombre d'une spiritualité déclinante qu'une laïcité récente écrase de tout son poids. Sous cet angle, la lumière jaune aux accents « sévère[s] » s'apparente à celle d'un 
coucher de soleil qui annonce la nuit. Dans ce tombeau à ciel ouvert, « l'ordre humain est, pour reprendre Bataille, [...] solidaire de l'ordre architectural, qui n'en est que le développement » (Bataille, 1929: 117). Et alors que la sécularisation des institutions laissait présager une liberté nouvelle pour l'individu, il semble que, dans l'économie balzacienne, elle se transforme en un espace d'errance pour l'âme et le cœur des hommes qui y sont soumis. Étrange invitation au voyage donc que cette entrée en matière, voyage vers le bas, mû par une verticalité qui aspire avec elle transcendance et joie. Qu'elle soit majestueuse comme celle des dômes, ou sépulcrale comme celle de la sinistre rue, dans chaque cas, la ligne descendante orthographie le sens du texte dans un glissement plurisémantique où la désolation géographique rencontre la ruine des symboles religieux, la déchéance sociale des individus et les afflictions humaines. Au sein d'un savant dédoublement graphique, la verticalité orchestre les discours muets des dispositifs topographique et idéologique. Par ailleurs, on voit aussi nettement que la sensibilité figurative du texte va de pair avec une sensibilité philosophique, voire métaphysique. Tout comme les institutions sont délaissées par Dieu, il semble que la rue Neuve-SainteGeneviève le soit par sainte Geneviève elle-même, patronne de Paris, puisque même le voyageur parisien qui s'aventure dans cet endroit s'y égare; c'est un fait qu'il se trouve là, dans le « quartier le plus horrible [et] le plus inconnu » de la ville (49). Aussi, loin des festivités et des mondanités parisiennes, le quartier SaintMarceau, et le récit dont il sera l'un des cadres principaux, se convertit pour notre passant en terre inconnue. Et pourtant, au milieu de ce miroir inversé du Paris fashionable et rieur, se terre peut-être sa vérité cachée et celle de tous les hommes : «Ah! sachez-le : ce drame n'est ni une fiction, ni un roman. All is true, il est si véritable, que chacun peut en reconnaître les éléments chez soi, dans son cœur peut-être. » (48-49.) Alors que généralement le flâneur jouit de " l'expérience de l'étrangeté [comme] d'un spectacle esthétique » (Stierle: 267), aucune sublimation de cet ordre n'atteint cette fois-ci le promeneur. Ce serait, selon Karlheinz Stierle, la proximité entre le Parisien et le quartier Saint-Marceau qui empêche ce genre d'expérience d'avoir lieu. Peutêtre est-ce le cas, mais nous croyons plutôt que cet étonnant voyageur prend physiquement conscience que le réel n'est plus subordonné au spirituel, les Catacombes sous ses pieds lui rappelant douloureusement sa finitude.

\section{Le cadre de bronze}

On sait que les incipits condensent les orientations des récits, aussi pour nous qui connaissons la fin tragique du père Goriot, «figure christique » (il n'hésite pas à se comparer à Dieu), et l'ascension fulgurante de Rastignac soutenue par des moyens peu vertueux, la marche perdue de ce passant malencontreux, prisonnier de la noirceur de la rue Neuve-Sainte-Geneviève et de l'ombre des dômes laïques, suggère que l'affranchissement d'une forme de transcendance expose l'être à un gouffre moral (les Catacombes) et à une esthétique tombale : 
La rue Neuve-Sainte-Geneviève surtout est comme un cadre de bronze, le seul qui convienne à ce récit, auquel on ne saurait trop préparer l'intelligence des couleurs brunes, par des idées graves; ainsi que, de marche en marche, le jour diminue et le chant du conducteur se creuse, alors que le voyageur descend aux Catacombes. Comparaison vraie! Qui décidera de ce qui est plus horrible à voir, ou des cœurs desséchés, ou des crânes vides? (49.)

Loin des « couleurs sanguinolentes des écrits modernes » (Balzac, 1979 [1831]: 54), sous-entendu de la littérature fantastique qui triomphe durant ces années 30 et dont se plaint amèrement l'écrivain dans sa «Préface » à La Peau de chagrin, celles du Père Goriot, dans l'incipit tout particulièrement préfèrent les tons mornes de la blessure morale.

Les habitués de La Comédie humaine reconnaitront certainement la tendance qui veut que, chez Balzac, I'histoire des mœurs - celle du père Goriot, d'Eugène de Rastignac, de madame Vauquer ou d'autres — débute avec le déficit des systèmes qui relèvent d'une transcendance. Et si l'auteur « écri[t] », comme il le dit lui-même dans son " Avant-propos », "à la lueur de deux Vérités éternelles : la Religion [et] la Monarchie » (Balzac, 1976 [1842]: 13), force est d'admettre que ces lueurs sont faibles, à l'image de celles que projettent les coupoles de la rue Neuve-Sainte-Geneviève. Peut-être participent-elles de l'esthétique du « siècle [que Vautrin qualifie de] mou » (225). Aussi pour en dégager des tableaux tranchés l'auteur juge-t-il nécessaire d'en fixer le cadre. On ne s'étonnera donc pas que les lignes verticales de la rue se cassent pour former un « cadre de bronze » qui enclôt des couleurs moins éclatantes que le sang, mais tout aussi funestes. En termes de nature de l'espace, le quartier de la pension Vauquer relève nettement du contenu, plus spécifiquement de la " prison » comme le souligne dès le début le narrateur (49); ceci se confirme quelques pages plus loin, lorsque les pensionnaires sont qualifiés de « forçats » (66). On le voit, le bronze et le carré s'intensifient mutuellement dans l'idée d'une frontière dure : dans cette combinaison, la ligne se meut en clôture. Si l'on suit la logique géométrique de l'incipit, on remarque que le cercle parisien (intra et extra muros) contient en son centre un quadrilatère (le cadre de bronze), figure ptoléméenne qui généralement invite à penser les changements de cadres, mais qui, dans le cas présent, sert à visualiser les changements de plans et de perspectives. En effet, entre le cercle de Paris et le cadre de bronze, Balzac déplace et promène son lecteur dans des univers différents et superposés, fortement liés les uns aux autres. De fait, d'une frontière à une autre ou du cercle au carré, la roue de la civilisation déroule la ligne horizontale du temps qui court, et celle verticale des religions mourantes strie le tableau d'une «scène de la vie privée $\underline{\underline{6}}$ ». L'ensemble trouve alors sa cohérence dans un même système de références, Paris, que métaphorise le mur d'octroi. Ainsi, le dynamisme du texte ne loge pas seulement dans la description des individus, des monuments ou des choses, il est soutenu également par ces variations graphiques — de la ligne droite à la ligne brisée, en passant par l'horizontale — qui s'effectuent à l'intérieur d'une structure giratoire (la ceinture parisienne) et où les sens, ceux de la pensée et du corps, 
peuvent être à la fois contradictoires et complémentaires, tout comme le sont les changements de focales du plus grand au plus petit —, les changements de perspectives — du plus haut au plus bas —, tout comme la ville rayonnante, centre du monde en ce dix-neuvième siècle débutant, accouche néanmoins, protégée par le silence des dômes, de geôles urbaines : ainsi la rue Neuve-Sainte-Geneviève.

Cette manière d'offrir une image morale de Paris, en en éclairant des coins sombres, est effectivement à l'œuvre dès l'incipit du roman. Le cadre de bronze, cadre généralement de petit format, illustre bien cette focalisation. Telle une étroite fenêtre dans la masse des «Études de mœurs », il s'ouvre sur les abîmes émotionnels et moraux des «âmes désolées » (Balzac, 2004 [1834]: 65) peuplant la maison Vauquer. Balzac scénarise la spatialité à l'aide d'un imaginaire fortement géométrique qui ordonne une vision de la société; c'est qu'architecture et idéologie sont farouchement mêlées dans l'univers balzacien, comme elles le seront à sa suite, «l'esprit de classe s'exprim[ant] dans la physionomie » des lieux, au sein du roman du second XIXe siècle (Stierle: 259). Que l'espace soit économiquement et socialement construit, la critique l'a largement démontré et analysé. Ce dont on parle moins cependant, c'est la congruence entre les lignes droites ou serpentines, entrelacées ou parallèles qui le traversent et le sens plus général du texte. Que l'on pense aux verticalités de l'incipit, aux ondulations de celui de La Peau de chagrin, aux lignes croisées qui ouvrent Le Colonel Chabert, aux ramifications généalogiques qui débutent $L a$ Vieille Fille ou à la ligne frontière de Sarrasine, dont Michel Serres a remarquablement montré la dissémination sémantique tout au long de la nouvelle (1989), celles-ci ou celles-là, toutes s'associent par analogie graphique au récit qu'elles initient. Dit autrement, c'est comme si une forme de figurabilité présidait aux significations du roman, comme si des schèmes graphiques plus ou moins obsédants (comme on parle de métaphores obsédantes) commandaient des destinées, découpaient des thèmes, traçaient des atmosphères, bref, dessinaient un imaginaire. Cette collaboration graphique entre le fond et les formes révèle que ce n'est pas seulement la représentation mimétique du réel qui importe, mais une représentation stratégique de lignes directionnelles qui rendent compte figurativement de la visée du roman, tout en servant aussi de passerelles signifiantes. De ce point de vue, l'incipit du Père Goriot est redoutable d'efficacité tant ces « lignes que l'on peut qualifier de section? ” (Brusatin: 56), celles-là même qui en anatomie servaient, à l'origine, à dévoiler l'âme derrière le muscle, œuvrent à révéler les différentes codifications du texte.

\section{Les lignes du drame}

Ce système linéaire est tout à fait patent dans les segments métanarratifs du roman. On se souvient que la ligne verticale descendante faisait référence au déclin des institutions religieuses, mais elle marque aussi celle du roman en tant que genre et plus spécifiquement celle du drame dont il est le support, comme nous l'avons déjà rapidement souligné. Ainsi, rappelons que dès le premier paragraphe le texte indique que c'est en termes 
de genre déconsidéré que le drame est présenté (47-48). La ligne descendante inscrite de facto dans la chute du dramatique « tombé » (48) hors de faveur accompagne donc figurativement une ligne évaluative qui fusionne informations commerciales et entreprise esthétique. Par ailleurs, le doute que jette le narrateur sur la possible incompréhension de l'histoire par un non-autochtone instaure une forme d'intimité avec le lecteur parisien, qui n'est pas n'importe quel lecteur, puisque savant de son expérience locale de la ville, et de ce fait seul capable de comprendre le drame se jouant dans ce livre. Derrière ces réserves topo- et ethnographiques, c'est toute une conception du drame et du roman, sensibles au lieu, aux faits divers, au quotidien et aux mœurs, que propose Balzac dès 1834, donc bien avant qu'il ne le théorise dans l'« Avant-propos » de 1842. Le cercle participe de cette conception; aussi celui du mur d'octroi de la ville de Paris ne vaut pas uniquement comme détail topographique. En tant que cercle fermé, il établit à la fois une frontière géographique, crée un club de lecteurs, ravive la ségrégation entre Parisiens et provinciaux, superpose données sociologiques et réception du livre, et révèle finalement à mots couverts des questions majeures qui sont aussi les enjeux du récit : par quels moyens un provincial peut-il et va-t-il saisir les rouages de la réussite parisienne? Par quels moyen un individu qui n'appartient pas à la coterie des bien-nantis peut-il un jour y participer? C'est donc, maillé à ce cercle, un problème de légitimité qui est révélé dès l'ouverture du Père Goriot : légitimité ethnographique (être ou ne pas être parisien), légitimité générique (être ou ne pas être un roman-8). La ligne circulaire prend donc en compte des phénomènes de frontières géographique et sociale, de rayonnement et d'esthétique, alors que la ligne verticale énonce essentiellement, dans cet incipit tout au moins, l'absence de transcendance (paradoxalement, pourrions-nous dire, tant la verticalité est liée à la transcendance dans notre imaginaire culturel) : la chute, la misère et la mort. On voit ainsi à quel point l'espace savamment et minutieusement décrit par le romancier obéit à une logique que nous pouvons qualifier de graphique, qui met en relation architecture, histoire, esthétisme, présupposés idéologiques et éthos social, et où le dessin de la ligne, pour mieux réfléchir ces différents points de vue du récit, se brise à angle droit ou se courbe : carré, cercle, chacune de ces figures ordonnant une combinatoire particulière qui complète en la nuançant ou en la confirmant la fonction descriptive et signifiante de la ligne, ici essentiellement descendante. Cette manière d'user de la ligne réaffirme que les schèmes géométriques sont des données avant tout anthropologiques (Lumbroso, 2004: 301) qui appartiennent à notre imaginaire culturel et qui servent de ponts entre différents niveaux textuels. II est remarquable en effet que la ligne, par sa seule direction, domestique l'espace, mais aussi les idées et les émotions. À l'intérieur d'un phénomène figural savant, comme l'examen de cet incipit le prouve, Balzac chorégraphie des récits, historiques et individuels, où les échappées axiales mêlent les lignes et les mots dans une organisation scripturale qui réconcilie vitalité du trait et profondeur du sens. 
1. Gustave Moreau dans ses Cahiers intimes, cité par GROJNOWSKI, Daniel. 2016. "Surréalisme. Vue d'ensemble », in Encyclopædia Universalis. Boulogne-Billancourt (France) : Encyclopædia Universalis, consulté le 14 novembre 2017.

2. L'extrait entier vaut d'être cité : «En entrant par le faubourg Saint-Marceau je ne vis que de petites rues sales et puantes, de vilaines maisons noires, l'air de malpropreté, de la pauvreté, des mendiants, des charretiers, des ravaudeuses, des crieuses de tisane et de vieux chapeaux. Tout cela me frappa d'abord à un tel point que tout ce que j'ai vu depuis à Paris de magnificence réelle n'a pu détruire cette première impression, et qu'il m'en est resté toujours un secret dégoût pour l'habitation de cette capitale. » (Rousseau: 187.)

3. Sur Paris dans La Comédie humaine, voir Jeannine Guichardet (1986a: 169-190; 1986b), mais aussi Isabelle Mimouni (1999).

4. [Nous soulignons.]

5. Sur l'histoire du Panthéon, voir Mona Ozouf (1984).

6. Dans le catalogue de La Comédie humaine de 1845 , Le Père Goriot est le $26^{\mathrm{e}}$ titre des « Scènes de la vie privée », elles-mêmes appartenant aux «Études de mœurs ».

7. Manlio Brusatin, à qui nous empruntons l'expression, l'emploie à caractériser les lignes tranchantes de la dissection anatomique (56).

8. On se souvient de l'affirmation : « ce drame n'est ni une fiction, ni un roman » (Balzac, 2004 [1834]: 49).

\section{Bibliographie}

BALZAC (DE), Honoré. 1976 [1842]. «Avant-propos à "La Comédie humaine" », dans La Comédie humaine, sous la direction de Pierre-Georges Castex. Paris : Gallimard, « Bibliothèque de la Pléiade », t. 1, p. 7-20.

BALZAC (DE), Honoré. 1979 [1831]. «La Peau de chagrin », dans La Comédie humaine, sous la direction de Pierre-Georges Castex. Paris : Gallimard, «Bibliothèque de la Pléiade », t. 10, p. 48-294.

Balzac (DE), Honoré. 2004 [1834]. Le Père Goriot, introduction, notes et dossier de Stéphane Vachon. Paris : Librairie générale française, «Classiques de poche », $443 \mathrm{p}$.

Batallle, Georges. 1929. «Architecture ». Documents, no 2, mai, p. 117.

BÉNICHOU, Paul. 1977. Le Temps des prophètes. Doctrines de l'âge romantique. Paris : Gallimard, «Bibliothèque des idées », $589 \mathrm{p}$.

Brusatin, Manlio. 2013 [1993]. Histoire de la ligne. Paris : Flammarion, « Champs art », 242 p.

GROJNOWSKI, Daniel. 2017 [2016]. «Surréalisme. Vue d'ensemble », dans Encyclopædia Universalis.

Boulogne-Billancourt (France) : Encyclopædia Universalis. <http://www.universalis-

edu.com/encyclopedie/surrealisme-vue-d-ensemble/>. Consultée le 14 novembre 2017.

a

Guichardet, Jeannine. 1986. Balzac, "archéologue" de Paris. Paris : SEDES, 498 p.

b

GuICHARDET, Jeannine. 1986. «Un jeu de l'oie maléfique. L'espace parisien du "Père Goriot"». L'Année

balzacienne, no 7, p. 169-189.

LumBroso, Olivier. 2004. Zola. La plume et le compas. La construction de l'espace dans "Les Rougon-

Macquart" d'Émile Zola. Paris : Champion, 320 p.

MımounI, Isabelle. 1999. Balzac illusionniste : les arts dans l'œuvre de l'écrivain Paris : Adam Biro, 127 p. 
Ozouf, Mona. 1984. «Le Panthéon. L'École normale des morts», dans Pierre NorA (dir.), Les lieux de mémoire. La République. Paris : Gallimard, « Bibliothèque illustrée des histoires », t. 1, p. 139-196.

Rousseau, Jean-Jacques. 1832 [1782]. Les Confessions. Paris : Imprimerie et fonderie de G. Doyen, t. 11, $395 \mathrm{p}$.

SerRes, Michel et Honoré BALZAC (DE). 1987. L'Hermaphrodite. Sarrasine sculpteur, précédé de Sarrasine. Paris : Flammarion, $161 \mathrm{p}$.

StIERLE, Karlheinz. 2001 [1993]. La capitale des signes, Paris et son discours, traduit par Marianne RocherJacquin, préface de Jean Starobinski. Paris : Maison des Sciences de l'Homme, 630 p. 